\title{
3D-Printed Composite Bone Bricks For Large Bone Tissue Applications
}

\author{
Evangelos Daskalakis ${ }^{1}$, Fengyuan Liu ${ }^{1}$, Anil A. Acar ${ }^{2}$, Edera-Elena Dinea $^{1}$, Glen Cooper ${ }^{1}$, \\ Andrew Weightman ${ }^{1}$, Bahattin Koç ${ }^{2}$, Gordon Blunn ${ }^{3}$, and Paulo Jorge Bártolo, ${ }^{1, *}$ \\ ${ }^{1}$ School of Mechanical, Aerospace and Civil Engineering, University of Manchester, Manchester, UK \\ ${ }^{2}$ Department of Manufacturing Engineering, Sabanci University, Instabul, Turkey \\ ${ }^{3}$ School of Pharmacy and Biomedical Sciences, University of Portsmouth, Portsmouth, UK
}

\begin{abstract}
This study investigates the use of low cost, customizable, biodegradable, polymer-ceramic composite porous structures (bone bricks) for large bone tissue regeneration. Different ceramic materials (hydroxyapatite (HA), $\beta$-tri-calcium phosphate (TCP) and Bioglass (45S5) were mixed with poly- $\varepsilon$-caprolactone (PCL). Bone bricks with different material compositions were produced using an extrusion-based additive manufacturing system. Produced bone bricks were morphologically and mechanically assessed. Results allowed to establish a correlation between scaffolds architecture and material composition and scaffolds performance. Reinforced scaffolds showed improved mechanical properties. Best mechanical properties were obtained with PCL/TCP bone bricks and topologies based on 38 double zig zag filaments and 14 spirals.
\end{abstract}

\section{Introduction}

Additive manufacturing is a very promising fabrication strategy for the production of threedimensional (3D) porous scaffods for the regeneration of damaged bones and other tissues [1-5]. These scaffolds must be infection resistant, biocompatible, biodegradable, custom made, cost-effective and modular, contributing to the establishment of a proper biomechanical environment that promotes tissue regeneration [6-8]. These characteristics can be obtained through a proper combination of scaffold design and suitable materials providing mechanical stability and promoting cell attachment, differentiation and proliferation [9-11].

Under an EPSRC/GCRF (Engineering and Physical Sciences Research Council/Global Challenges Re-search Fund) project entitled "Bone Bricks: Low cost effective modular osseointegration prosthetics for large bone loss surgical procedures" we are developing a novel low cost osseointegrated modular prosthetic solution to treat large bone loss injuries $(20 \mathrm{~cm})$ to enable limb salvage. The immediate application is to treat Syrian refugees who have been displaced to Turkey, but it can be used for injuries made from conflict or natural disasters. The project proposes to build on the current treatment of external fixation but with the addition of an engineered internal prosthetic implant to improve patient outcomes, avoid painful limb lengthening and reduce recovery time. A patient specific prosthetic to fill the bone lost due to injury is being developed using biodegradable and biocompatible modular

${ }^{*}$ Corresponding author: paulojorge.dasilvabartolo@manchester.ac.uk 
pieces (bone bricks) from a pallet of shapes and sizes that fit together in a lego-like way to form the prosthesis. The assembled prosthesis will create a hollow cage which will be filled with an infection prevention paste containing calcium sulphate and polymeric microbeads encapsulating antibiotics (Fig. 1). This paper presents preliminary results considering anatomical designed bone bricks produced using different architectures and material composition. Produced structures are morphologically and mechanically investigated.

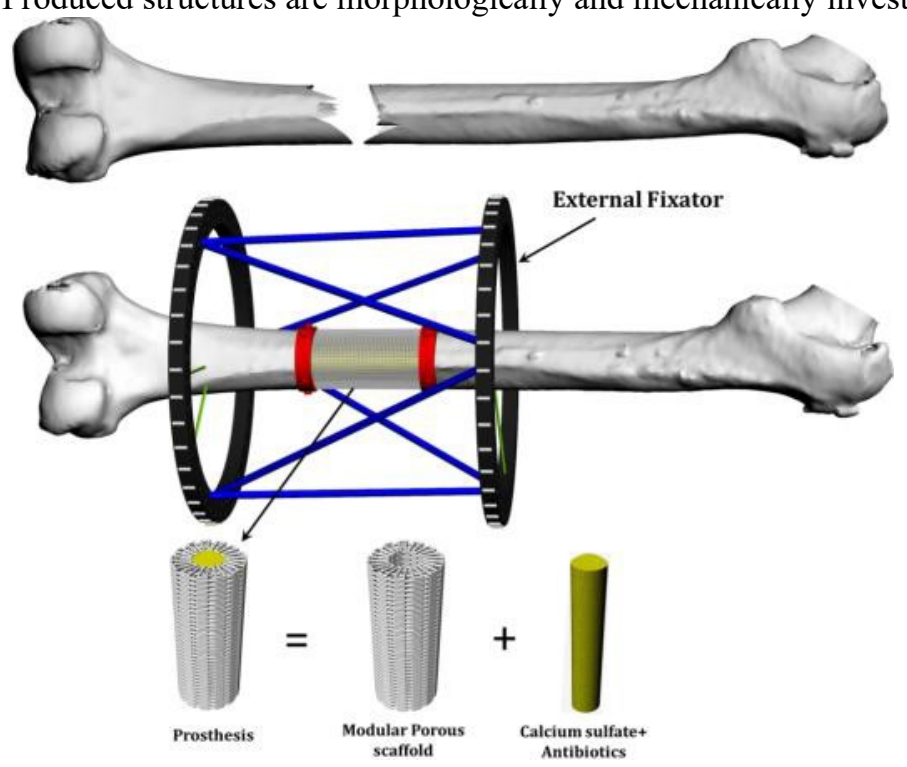

Fig. 1. Bone bricks approach for large bone loss treatment.

\section{Materials and Methods}

\subsection{Materials}

Polycaprolactone (PCL) (CAPA 6500, Mw=50000Da), a semi-crystalline linear aliphatic biopolymer, was supplied by Perstorp Caprolactones (Cheshire, UK) in the form of pellets. Hydroxyapatite $(\mathrm{HA})\left(\mathrm{Mw}=502.31 \mathrm{r} / \mathrm{mol}, \mathrm{MP}=1100^{\circ} \mathrm{C}\right)$ was supplied by Sigma-Aldrich (St. Loius, USA) in a nanopowder form $(<200 \mathrm{~mm}$ particles size), $\beta$-tri-calcium phosphate (TCP) $\left(\mathrm{Mw}=310.18 \mathrm{r} / \mathrm{mol}, \mathrm{MP}=1391{ }^{\circ} \mathrm{C}\right)$ was supplied by Sigma-Aldrich (St. Loius, USA) in a nanopowder form $(<200 \mathrm{~mm}$ particles size) and Bioglass (45S5), with a composition of $45 \mathrm{wt} \% \mathrm{SiO}_{2}, 24.5 \mathrm{wt} \% \mathrm{CaO}, 24.5 \mathrm{wt} \% \mathrm{Na}_{2} \mathrm{O}$, and $6 \mathrm{wt} \% \mathrm{P}_{2} \mathrm{O}_{5}$, was supplied by CeraDynamics Ltd. James Kent Group (Stoke, UK) in nanopowder form $(<0.005 \mathrm{~mm}$ particles size). PCL composite blends containing different bioceramic contents $(20 \mathrm{wt} \% \mathrm{HA}, 20 \mathrm{wt} \% \mathrm{TCP}$ and $20 \mathrm{wt} \%$ Bioglass) were produced by melt blending. Briefly, PCL pellets were melted at 150 ${ }^{\circ} \mathrm{C}$ in a porcelain bowl before adding the ceramic material. Composite materials were mixed around 1 hour to ensure a uniform distribution of the ceramics in the polymer mix.

\subsection{Scaffolds Production}

Bone bricks were produced using the screw-assisted extrusion based additive manufacturing 3D Discovery system (RegenHU, Switcherland). A computational geometry-based algorithm, with data collected from anthropometric measurements by surgeons in Turkey was used to create a continuous path planning algorithm, using zig-zag (25 and 38 double 
filaments) and spiral like patterns (9 and 14 filaments) to produce four groups of bone bricks with overall porosity varying between $52 \%$ and $68 \%$ (Table 1 ). The process parameters used for the production of the scaffolds were: melting temperature of $90{ }^{\circ} \mathrm{C}$, deposition velocity of $18 \mathrm{~mm} / \mathrm{s}$ and screw rotation velocity of $14 \mathrm{rpm}$. The diameter of the needle was $0.33 \mathrm{~mm}$.

Table 1. Anthropometric-based geometries and different path planning strategies considered to produce bone bricks with different porosities.

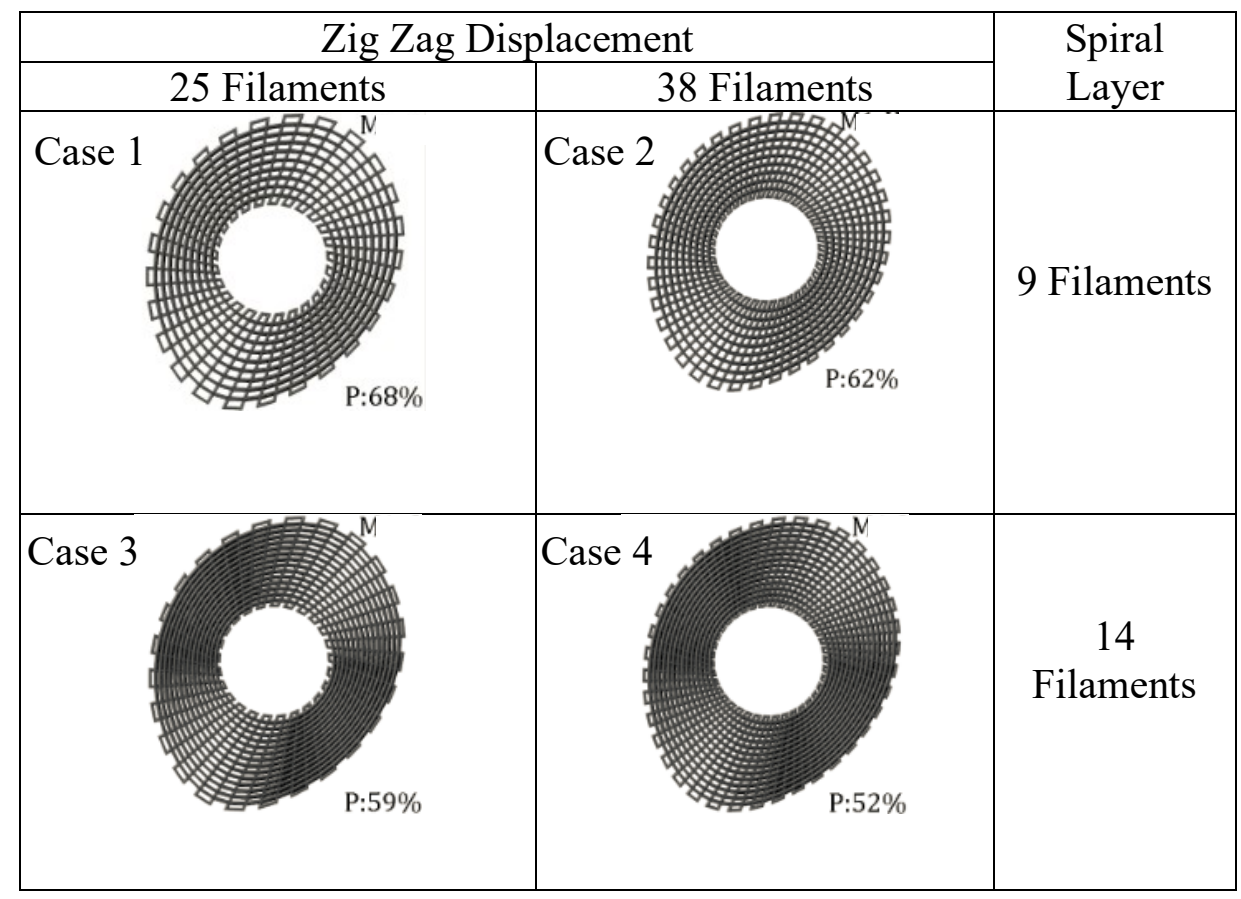

\subsection{Morphological Characterization}

The morphology of printed scaffolds was investigated using the Scanning Electron Microscopy (SEM) FEI ESEM Quanta 250 (FEI Company, United States) at an accelerated voltage of $15 \mathrm{kV}$. EMITECH K550X sputter coater (Quorum Technologies, UK) was used for coating the structures (gold coating) prior imaging. The obtained images were processed by Image J (NIH, USA) allowing to determine the pore size (PS) and the filament width (FW). For each scaffold 10 measurements were considered to obtain the average and standard deviation.

\subsection{Mechanical Characterization}

Compression tests were performed on the INSTRON 3344 (Instron, UK) in dry state with a $2 \mathrm{kN}$ load cell and a displacement rate of $0.5 \mathrm{~mm} / \mathrm{min}$, according to the ASTM D695-15. The Bluehill Universal software (Instron, UK) was used to collect the data and to determine compression modulus.

\section{Results}




\subsection{Morphological Analysis}

Fig. 2 shows high magnification SEM images of both top view and cross-section view of printed bone bricks considering as an example case 2 and all material compositions. Pore size and filament width values are presented in Table 2. Results show that pore size decreases and filament width increase by increasing the bioceramic content. Moreover, for the same configuration and level of reinforcement filament width is higher (lower pore size) in HA bone bricks than in TCP and Bioglass scaffolds. The pore size also decreases by increasing the number of spiral filaments.

Table 2. Morphological characteristics of bone bricks considering different material compositions.
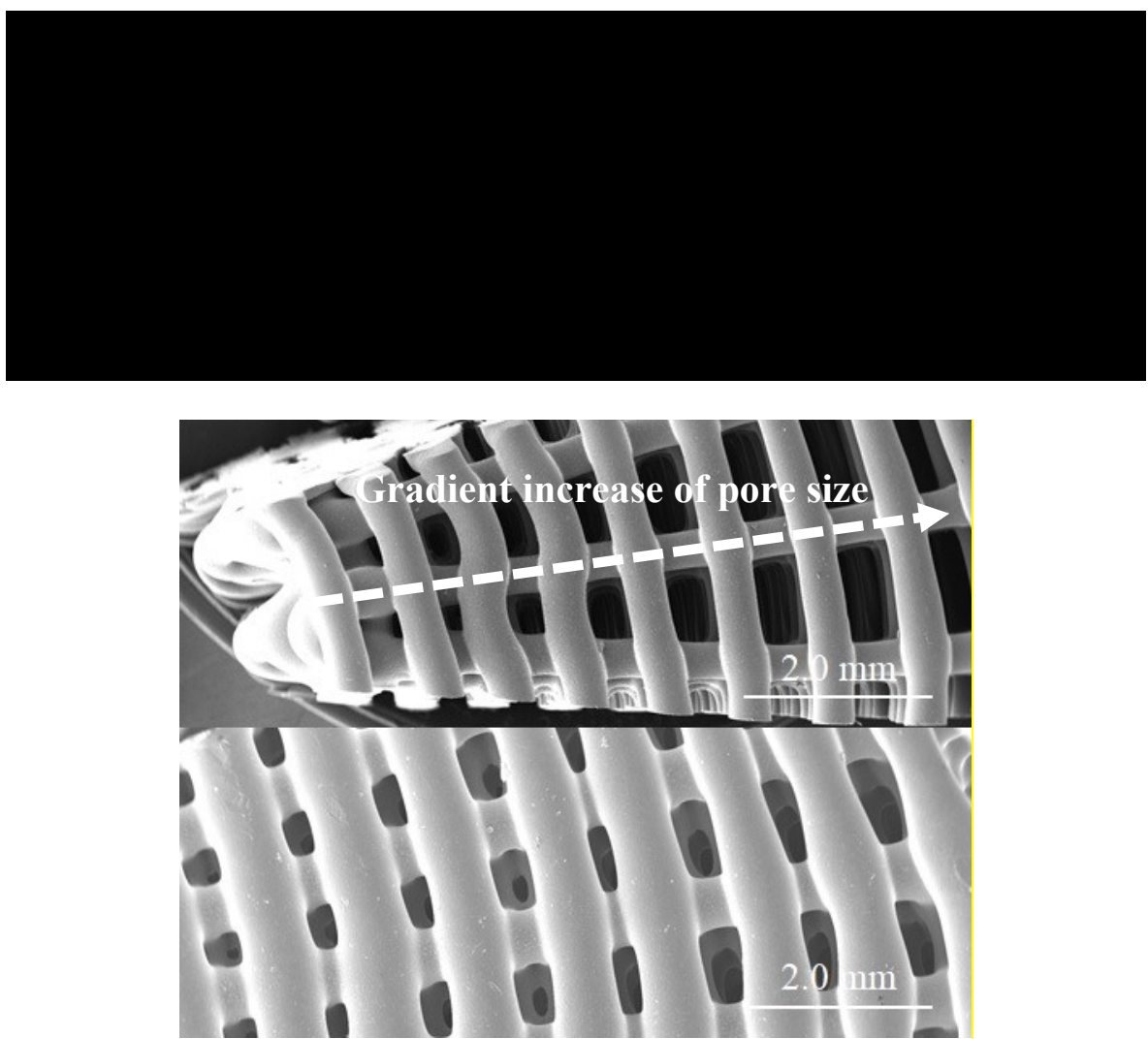

Fig. 2. SEM images of the top view of $20 \mathrm{wt} \%$ HA scaffolds with different architectures (A) case 1 and (B) case 4 .

\subsection{Mechanical Analysis}

As shown in Fig. 3, the mechanical behavior of the bone bricks strongly depends on the architecture and ceramic content. For the same architecture, the compressive modulus increases by increasing the ceramic content and for the same level of reinforcement the compressive modulus is higher in bone bricks containing TCP than HA and Bioglass. For scaffolds with the same material composition and the same number of double layers, compressive modulus increases by increasing the number of spiral filaments. This can be explained by the overall decrease in porosity. Results also show that by controlling the number of double and spiral filaments it is possible to create scaffolds with compressive modulus in the trabecular region and presenting much higher values than previously reported for standard regular square scaffolds [12]. 

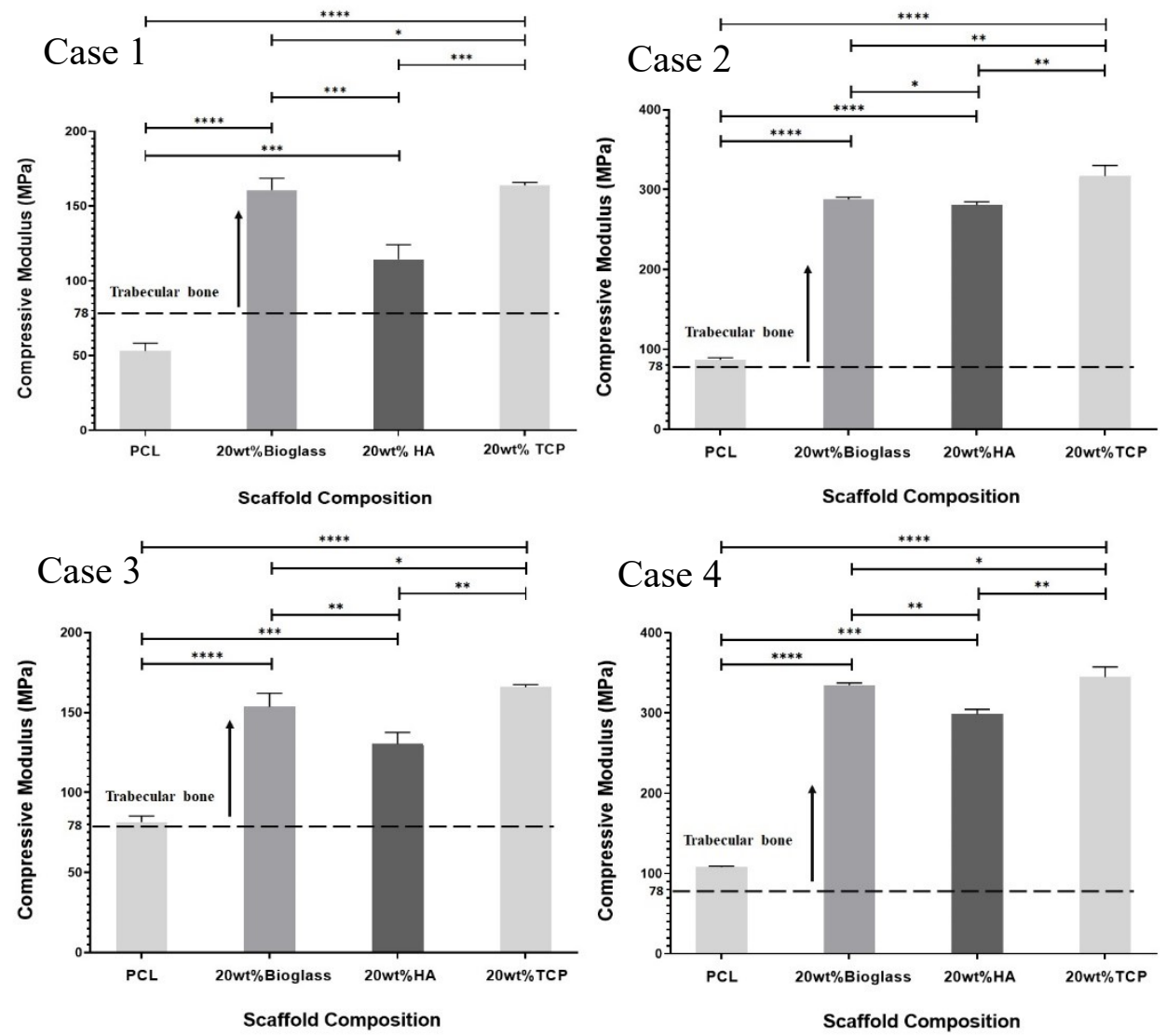

Fig. 3. Compressive modulus as a function of bone brick architecture and material composition. *Statistical evidence $(\mathrm{p}<0.05)$ analyzed by one-way ANOVA, and Tukey post-test.

\section{Conclusion}

This paper investigates the effects of bone bricks architectures and materials composition on the morphological and mechanical properties of printed structures, which were fabricated according to anthropometric measurements. The results show that scaffolds with complex architectures mimicking the patient bone structure were successfully produced using the screw-assisted extrusion based additive manufacturing. Moreover, results show that pore size decreases and filament width increases by adding ceramics into the polymeric matrix, while for the same configuration and level of reinforcement, filament width is higher (lower pore size) in PCL/HA scaffolds than in PCL/TCP and PCL/Bioglass scaffolds. Results also show that scaffolds produced using architecture 2 show improved mechanical properties compared to the other architectures. The addition of ceramics (Bioglass, HA and TCP) enhances the mechanical properties of all bone bricks configurations. Mechanical proprieties increase, for the same concentration of ceramic reinforcements, as follows: PCL/TCP, PCL/Bioglass and PCL/HA.

This project has been supported by the University of Manchester and the Engineering and Physical Sciences Research Council (EPSRC) of the UK, the Global Challenges Research Fund (CRF), grant number EP/R01513/1. 


\section{References}

1. F. Melchels, M. Domingos, T. Klein, J. Malda, P. Bartolo, D. Hutmacher, Pr. in Pol. Sc., 37, 1079 (2012)

2. R. Katari, A. Peloso, G. Orlando, Adv. in Sur., 48, 137 (2014)

3. A. Mao, D. Mooney, Proc. of the Nat. Acad. of Sc., 112, 14452 (2015)

4. J. Lee, W. Ng, W. Yeong, App. Phys. Rev. 6, 011307 (2019)

5. W. Ng, C. Chua, Y. Shen, Progr. In Pol. Sc., 97, 101145 (2019)

6. P. Bartolo, J. Kruth, J. Silva, G. Levy, A. Malshe, K. Rajurkar, M. Mitsuishi, J. Ciurana, M. Leu, CIRP AN, 61, 635 (2012)

7. F. Liu, B. Huang, S. Hinduja, P. Bartolo, Biocer. And Biocomp.: From Res. To Clin. Pract., 17 (2019)

8. P. Lichte, , H. Pape, T. Pufe, P. Kobbe, H. Fischer, Inj., 42, 569 (2011)

9. C. Vyas, R. Pereira, B. Huang, F. Liu, W. Wang, P. Bartolo, Cur. Opin. in Biom..Eng., 2, 1 (2017)

10. B.Guillotin, F. Guillemot, Tren. in Biot., 29, 183 (2011)

11. S. Murphy, A. Atala, Nat. Biot., 32, 773 (2014)

12. B. Huang, G. Caetano, C. Vyas, J. Blaker, C. Diver, P. Bártolo, Mat., 11, 129 (2018) 